Original research article

\title{
The importance of atrial fibrillation and selected echocardiographic parameters for the effectiveness and safety of thrombolytic therapy in patients with stroke
}

\author{
Anetta Lasek-Bal ${ }^{a, *}$, Teresa Kowalewska-Twardela ${ }^{b}$, \\ Aldona Warsz-Wianecka ${ }^{a}$, Przemysław Puz ${ }^{a}$, Aleksandra Krzan ${ }^{c}$, \\ Aleksandra Cieślik ${ }^{a}$

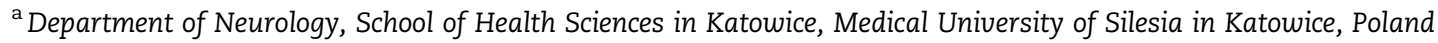 \\ ${ }^{\mathrm{b}}$ Medical University of Silesia Hospital No. 7 Upper Silesian Medical Centre, Katowice, Poland \\ ${ }^{\mathrm{c}}$ Department of Neurology, Medical University of Silesia Hospital No. 7 Upper Silesian Medical Centre, Katowice, \\ Poland
}

\section{A R T I C L E I N F O}

Article history:

Received 28 September 2016

Accepted 4 September 2017

Available online 19 September 2017

Keywords:

Stroke

Thrombolysis

Atrial fibrillation

Echocardiography

\begin{abstract}
A B S T R A C T
Background: The efficacy and safety of thrombolytic therapy in stroke depend on multiple factors. The aim of this study was to evaluate the significance of atrial fibrillation the prognosis in terms of the functional status in patients with stroke treated with intravenous thrombolysis. An additional aim was also to assess the potential significance of reduced ejection fraction (EF) and enlarged left atrium (LA) of the heart for the prognosis in patients with stroke who underwent thrombolytic therapy.

Methodology: A prospective study involved enrollment of 222 patients, mean age of 72 years with first-in-life ischemic stroke. In all participants there were realized procedures as follows: neurological status before administering rt-PA (NIHSS), selected echocardiographic parameters, functional status on the 14th day from the onset (mRankin scale) and analysis the bleeding events.

Results: Atrial fibrillation was significantly more frequent in women than men; females had higher CHA2DS2VASc scores and heavier neurological conditions on day 1 of stroke. Two independent factors for poor prognosis (3-5 points by mRankin) were found: the NIHSS score and the CHA2DS2VASc score $\geq 3$. We identified 2 independent factors for death within 14 days from the onset: the result by NIHSS and the EF. The NIHSS score turned out to be the only independent predictor of hemorrhage during hospitalization: RR 1.19; CI [1.06-1.33]; $p=0.003 ; p$ for model $=0.0025$.

Conclusions: The presence of atrial fibrillation worsens the patient's prognosis in terms of the functional status and survival during the acute period of stroke in patients treated with intravenous thrombolysis.
\end{abstract}

\footnotetext{
* Corresponding author at: Department of Neurology, School of Health Sciences in Katowice, Medical University of Silesia in Katowice, 40735 Katowice, ul. Ziołowa 45/47, Poland.

E-mail address: alasek@gcm.pl (A. Lasek-Bal). http://dx.doi.org/10.1016/j.pjnns.2017.09.001

0028-3843/@ 2017 Published by Elsevier Sp. z o.o. on behalf of Polish Neurological Society.
} 
Higher NIHSS and CHA2DS2VASc scores and reduced EF in patients with stroke treated with thrombolysis are the predictors of unfavorable short-term prognosis.

() 2017 Published by Elsevier Sp. z o.o. on behalf of Polish Neurological Society.

\section{Background}

The efficacy and safety of thrombolytic therapy in stroke depend on multiple factors. Although the potential importance of atrial fibrillation (AF) for the above parameters has been the subject of several studies, a disparity between their results prevents us from formulating a clear conclusion. It is likely that both the type of atrial fibrillation and coexisting structural and functional heart disorders accompanying arrhythmia may play a role regardless of the electromechanical and hemodynamic effects associated with AF. Given the prevalence of AF among patients with stroke, one should define the meaning of arrhythmia for the effects of thrombolytic therapy, as it may affect the selection of patients who could obtain the best benefits from such treatment.

The aim of this study was to evaluate the significance of AF for the prognosis in terms of the functional status in patients with stroke treated with intravenous thrombolysis. An additional aim was also to assess the potential significance of reduced ejection fraction (EF) and enlarged left atrium (LA) of the heart for the prognosis in patients with stroke who underwent thrombolytic therapy.

\section{Methodology}

A prospective study (period: 2014-2015) involved enrollment of 222 patients, mean age of 72 years (39-89; 107 women, 155 men); the subjects were patients with first-in-life stroke diagnosed by the WHO's clinical criteria, and an ischemic focus in the brain present in neuroimaging (CT and/or MRI of the head) [1]. All the subjects received recombinant tissue plasminogen activator (rt-PA) intravenous within $4.5 \mathrm{~h}$ from the disease onset, which was in accordance with the eligibility criteria for thrombolytic therapy [2]. The study excluded the patients who underwent endovascular treatment due to cerebral stroke - the aim of the study was to evaluate the importance of selected parameters for the prognosis of patients treated homogeneously (without the effect of general anesthesia used in approximately half of the patients given endovascular treatment in our center).

All the patients included in the study were examined in terms of the following:

- the patient's age at the time of first-ever ischemic stroke;

- presence of atrial fibrillation (valvular and non-valvular); AF due to reasons other than valvular heart disease was considered as non-valvular atrial fibrillation;

- presence of conditions/comorbidities such as arterial hypertension, diabetes and lipid disorders; $>70 \%$ of atherosclerotic carotid artery stenosis (ipsilaterally to the ischemic focus in the brain);
- neurological status evaluated before administering rt-PA according to NIHSS (National Institutes of Health Stroke Scale) [3];

- anticoagulant therapy conducted before the stroke onset;

- result by the CHA2DS2VASc scale;

- selected echocardiographic parameters: the presence of thrombus in the left atrium, reduced $\mathrm{EF}(<55 \%)$ and enlarged left atrium (LA $>3.8 \mathrm{~cm}$ for women and $>4.0 \mathrm{~cm}$ for men relative to body surface area);

- intracranial bleeding

- extracranial bleeding: from the nasal cavities, gastrointestinal tract, genital and urinary tract or from other areas requiring substitution with 2 units of packed red blood cells;

- functional status on the 14th day from the onset according to mRankin scale [4].

The diagnosis of hypertension was consistent with the recommendation by the Polish Society of Cardiology [5]; diabetes mellitus was diagnosed according to the criteria by the Polish Diabetes Association (2013) [6]; dyslipidemia was defined as total cholesterol serum level $>200 \mathrm{mg} / \mathrm{dl}$ ( $>5.18 \mathrm{mmol} / \mathrm{l})$; or LDLcholesterol serum level $>100 \mathrm{mg} / \mathrm{dl}(2.59 \mathrm{mmol} / \mathrm{l})$; or $\mathrm{HDL}$ cholesterol serum level $<35 \mathrm{mg} / \mathrm{dl}$ (0.91 mmol/l); or triglycerides serum level $>135 \mathrm{mg} / \mathrm{dl}(1.53 \mathrm{mmol} / \mathrm{l})$.

The degree of common carotid artery stenosis and/or internal carotid stenosis was rated according to the NASCET criteria [7].

Patients were divided into 2 groups depending on the presence of $\mathrm{AF}$ (present vs. absent) and depending on the CHA2DS2VASc result ( $<3$ vs. $\geq 3$ points); results were compared in parallel groups.

The type of intracranial bleeding was determined according to the ECASS I and II definition (European Cooperative Acute Stroke Study) $[8,9]$.

Multivariate analysis was performed in order to determine independent factors for unfavorable prognosis defined as obtaining 3-5 points on mRankin scale by a patient on their 14th day after stroke or as the patient's death within 14 days of onset. The analysis included the following parameters: age (also in subgroups: $<65$ years and $\geq 65$ years), sex (including subgroups: $<65$ years and $\geq 65$ years), arterial hypertension, diabetes, atrial fibrillation (valvular and non-valvular AF), lipid disorders, stenosis of the carotid artery $>70 \%$, NIHSS, CHA2DS2VASc $\geq 3, \mathrm{EF}<55 \%$, enlargement of LA and bleeding during 14-days hospitalization.

\section{Results}

Demographic and selected clinical patients' parameters are presented in Table 1. 


\section{Table 1 - Characteristics of patients.}

\begin{tabular}{ll} 
Patients, $n$ & 222 \\
Age, mean (median, range) & $69.7 \pm 10.8(72 ; 39-89)$ \\
F/M & $107 / 115(48.1 \% / 51.9 \%)$ \\
Arterial hypertension, $n(\%)$ & $175(78.8)$ \\
Diabetes mellitus, $n(\%)$ & $74(33.3)$ \\
Atrial fibrillation, $n(\%)$ & $81(36.4)$ \\
de novo, $n(\%)$ & $8(9.8)$ \\
Valvular AF & $42(51.8)$ \\
Carotid artery stenosis $>70 \%, n(\%)$ & $67(30.1)$ \\
Lipid disorders, $n(\%)$ & $53(23.8)$ \\
Past and documented myocardial & $37(16.66)$ \\
$\quad$ infarction, $n$ (\%) & \\
CHA2DS2VASc, mean (range) & $4.05(2-9)$ \\
NIHSS, mean (range) & $8.46(4-25)$ \\
Previous anticoagulant therapy, $n(\%)$ & $32(14.5)$ \\
\hline
\end{tabular}

F - female, M - male, NIHSS - National Institutes of Health Stroke Scale.

Table 2 - Comparison of selected parameters between the group of patients with AF and patients without AF.

\begin{tabular}{|c|c|c|c|}
\hline & $\begin{array}{c}\mathrm{AF}^{-} \\
n=141\end{array}$ & $\begin{array}{c}\mathrm{AF}^{+} \\
n=81\end{array}$ & $p$ \\
\hline $\mathrm{F}$ & 59 (41.8\%) & $48(59.3 \%)$ & $0.0124^{*}$ \\
\hline Age & $69 \pm 12.2$ & $71 \pm 9.4$ & $0.782^{\#}$ \\
\hline EF & $52.7 \pm 7.2$ & $44.8 \pm 9.0$ & $0.0000^{\#}$ \\
\hline LA size & $40.3 \pm 3.0$ & $42.8 \pm 3.6$ & $0.0000^{\#}$ \\
\hline CHA2DS2VASc & $3.7 \pm 1.8$ & $4.4 \pm 1.9$ & $0.0025^{+}$ \\
\hline CHA2DS2VASc $\geq 3$ & 75 (53.2\%) & $61(75.3 \%)$ & $0.0011^{+}$ \\
\hline NIHSS & $7.3 \pm 4.9$ & $9.6 \pm 5.5$ & $0.0016^{+}$ \\
\hline mRankin & $2.8 \pm 1.9$ & $3.7 \pm 2.0$ & $0.0012^{+}$ \\
\hline mRankin 3-5 & $79(56.0 \%)$ & $58(71.6 \%)$ & $0.0215^{\circ}$ \\
\hline Death & $10(7.1 \%)$ & $17(21.0 \%)$ & $0.0023^{*}$ \\
\hline $\begin{array}{l}\text { Iatrogenic hemorrhage } \\
\text { including }\end{array}$ & $3(2.1 \%)$ & $6(7.4 \%)$ & $0.0549^{*}$ \\
\hline intracranial bleeding & $3(2.1 \%)$ & $2(2.4 \%)$ & $0.0487^{*}$ \\
\hline \multicolumn{4}{|c|}{$\begin{array}{l}\text { F- female, } \mathrm{AF}^{-}-\text {patients without atrial fibrillation, } \mathrm{AF}^{+}-\text {patients } \\
\text { with atrial fibrillation, } \mathrm{EF}-\text { ejection fraction, LA - left atrium, NIHSS } \\
\text { - National Institutes of Health Stroke Scale } \\
\text { Chi2. } \\
\text { \# Student test. } \\
+ \text { U Mann-Whitney test. }\end{array}$} \\
\hline
\end{tabular}

A comparison between the patients with atrial fibrillation and those with no arrhythmia found a number of statistically significant differences in demographic, echocardiographic and clinical parameters, as shown in Table 2.

In the group of patients with CHA2DS2VASc score $\geq 3$, there were significantly more females, patients with atrial fibrillation, including valvular AF, and patients with unfavorable clinical and echocardiographic parameters compared to patients with CHA2DS2VASc score $<3$, as shown in Table 3. Differences in the incidence of hemorrhage during hospitalization, and death within 14 days of the onset of stroke, were not statistically significant.

Atrial fibrillation was significantly more frequent in women than men; females had higher CHA2DS2VASc scores and heavier neurological conditions on day 1 of stroke. There were no significant differences in the consequences of stroke (according to mRankin) in a 14-day observation (Table 4).

Two independent factors for poor prognosis (3-5 points by mRankin) were found: the NIHSS score and the CHA2DS2VASc score $\geq 3$ (Table 5)

We identified 2 independent factors for death within 14 days from the onset: the result by NIHSS and the EF (Table 6).

The NIHSS score turned out to be the only independent predictor of hemorrhage during hospitalization: RR 1.19; CI [1.06-1.33]; $p=0.003 ; p$ for model $=0.0025$.

\section{Discussion}

Age, diabetes and stroke within the preceding 3 months are recognized factors that modify the effectiveness of rt-PA in acute cerebral stroke. Previous observations do not allow for a clear identification of the importance of AF for the effect of thrombolytic therapy, especially in the acute phase of disease. Results of several studies suggest a worse clinical effect and lower safety of rt-PA application in patients with AF; however, it is difficult to draw consistent conclusions due to the differences in study protocols [10-13]. It has been indicated that there was a lower percentage for the recanalization of arteries in the patients with AF treated with rt-PA compared to

Table 3 - Comparison of selected parameters between the group of patients with CHA2DS2VASc score $<3$ and patients with CHA2DS2VASc score $\geq 3$.

\begin{tabular}{|c|c|c|c|}
\hline & $\begin{array}{c}\text { CHA2DS2VASc }<3 \\
n=86\end{array}$ & $\begin{array}{c}\text { CHA2DS2VASc } \geq 3 \\
n=136\end{array}$ & $p$ \\
\hline $\mathrm{F}$ & $31(36.0 \%)$ & 76 (55.9\%) & $0.0040^{\circ}$ \\
\hline Age & $68 \pm 11.9$ & $70 \pm 10.1$ & $0.692^{\#}$ \\
\hline $\mathrm{AF}$ & 20 (23.3\%) & 61 (44.9\%) & 0.0011 \\
\hline Valvular AF & $6(7.0 \%)$ & 36 (26.5\%) & 0.0003 \\
\hline $\mathrm{EF}$ & $52.0 \pm 7.2$ & $48.4 \pm 9.3$ & $0.0021^{*}$ \\
\hline LA size & $40.5 \pm 3.6$ & $41.6 \pm 3.4$ & $0.0143^{\prime}$ \\
\hline NIHSS & $7.0 \pm 5.3$ & $8.9 \pm 5.1$ & 0.0037 \\
\hline Rankin & $2.5 \pm 1.9$ & $3.5 \pm 1.9$ & 0.0005 \\
\hline Rankin 3-5 & $41(47.7 \%)$ & $96(70.6 \%)$ & 0.0006 \\
\hline Death & $6(7.0 \%)$ & 21 (15.4\%) & 0.0602 \\
\hline Iatrogenic hemorrhage & $5(5.8 \%)$ & $4(2.9 \%)$ & 0.2904 \\
\hline \multicolumn{4}{|c|}{$\begin{array}{l}\text { F - female, AF - atrial fibrillation, EF - ejection fraction, LA - left atrium, NIHSS - National Institutes of Health Stroke Scale. } \\
\text { Chi2. } \\
\text { \# Student test. } \\
+ \text { U Mann-Whitney test. }\end{array}$} \\
\hline
\end{tabular}


Table 4 - Comparison of selected parameters depending on the patient's sex.

\begin{tabular}{llll} 
& \multicolumn{1}{c}{$\mathrm{M}$} & \multicolumn{1}{c}{$\mathrm{F}$} & $p$ \\
& $n=115$ & $n=107$ & \\
\hline AF & $33(28.7 \%)$ & $48(44.9 \%)$ & $0.0124^{*}$ \\
Age & $68 \pm 4.4$ & $72 \pm 2.6$ & $0.167^{\#}$ \\
Valvular AF & $17(14.8 \%)$ & $25(23.4 \%)$ & $0.1028^{*}$ \\
EF & $48.7 \pm 8.9$ & $50.9 \pm 8.4$ & $0.0608^{\#}$ \\
LA size & $41.1 \pm 3.2$ & $41.3 \pm 3.8$ & $0.6921^{\#}$ \\
CHA2DS2VASc & $3.6 \pm 1.6$ & $4.3 \pm 2.0$ & $0.0093^{+}$ \\
CHA2DS2VASc $\geq 3$ & $60(52.2 \%)$ & $76(71.0 \%)$ & $0.0040^{*}$ \\
NIHSS & $7.3 \pm 5.1$ & $9.0 \pm 5.3$ & $0.0073^{+}$ \\
mRankin & $2.9 \pm 1.9$ & $3.3 \pm 2.0$ & $0.2060^{+}$ \\
mRankin 3-5 & $67(58.3 \%)$ & $70(65.4 \%)$ & $0.2728^{*}$ \\
Death & $12(10.4 \%)$ & $15(14.0 \%)$ & $0.4143^{*}$ \\
Iatrogenic hemorrhage including & $6(5.2 \%)$ & $3(2.8 \%)$ & $0.3622^{*}$ \\
Intracranial bleeding & $3(2.6 \%)$ & $2(1.9 \%)$ & $0.210^{*}$ \\
\hline AF - atrial fibrillation, EF - ejection fraction, LA - left atrium, NIHSS \\
- National Institutes of Health Stroke Scale. & \\
* Chi2. & & & \\
\# Student test. & & & \\
+ U Mann-Whitney test. & & & \\
\hline
\end{tabular}

Table 5 - Independent factors for poor prognosis (3-5 points by mRankin).

\begin{tabular}{lccc} 
& $\mathrm{RR}$ & $p$ for factor $p$ for model \\
\hline NIHSS & 1.30 CI [1.19-1.42] & 0.0000 & 0.0000 \\
CHA2DS2VASc $\geq 3$ & 2.11 CI [1.11-4.02] & 0.0220 & \\
\hline \multicolumn{4}{l}{ NIHSS - National Institutes of Health Stroke Scale. } \\
\hline
\end{tabular}

the patients without $A F$ [10]. In the NINDS study, patients with AF obtained worse scores than patients without arrhythmia (OR for a more favorable outcome: 0.57; 95\% CI; 0.38-0.86) [14]. Analysis of the ECASS III trial results also indicates such a trend; however, there was no statistically significant difference (OR: 0.68; 95\% CI; 0.30-1.55) [15]. On the contrary, the results of the meta-analysis entitled the Virtual International Stroke Trials Archive (VISTA) do not confirm the negative impact of AF on the effect of thrombolytic therapy [16].

In the present study, the presence of AF was significantly more often associated with unfavorable prognosis in terms of the functional status and the effect of death within the first 2 weeks of the onset of stroke. It is worth emphasizing that iatrogenic intracranial bleeding did not modify the results in a statistically significant manner.

Perhaps the group of patients with AF should be regarded as a relatively heterogeneous due to the AF type; that would explain the disparity between the results obtained by the authors. It has been recently stressed that compared to patients with permanent atrial fibrillation a significantly better prognosis relates to patients with paroxysmal AF [17]. It has been suggested that the duration of arrhythmia is significant for the formation of thrombus and embolic risk. Moreover, electromechanical and hemodynamic effect accompanying AF are probably not the only parameters potentially modifying the effect of therapy in this group of patients. Structural and functional heart abnormalities may also play an important role. In the present study, significantly more often it was patients with AF who had reduced ejection fraction, which
Table 6 - Independent factors for death within 14 days from the onset.

\begin{tabular}{lccc} 
& RR & $p$ for factor & $p$ for model \\
\hline NIHSS & 1.19 CI [1.10-1.29] & 0.0000 & 0.0000 \\
EF & 0.94 CI [0.89-0.98] & 0.0087 & \\
\hline
\end{tabular}

NIHSS - National Institutes of Health Stroke Scale, EF - ejection fraction.

reflects the dysfunction of the left ventricle and promotes the formation of thrombi. Based on the analysis of multiple variables, it was demonstrated that the presence of moderate to severe LV systolic dysfunction observed in two-dimensional echocardiography is an independent echocardiographic factor for acute embolic incident $[18,19]$. A significant decrease in EF was demonstrated in patients with cardiogenic and cryptogenic stroke [20]. The present study also revealed a negative impact of reduced EF on the course of stroke; low EF reduces the benefits of thrombolytic therapy. In the present study, significantly more often it was patients with AF who had an enlarged left atrium. As demonstrated, cardiac systolic or diastolic dysfunction, arterial hypertension, diabetes, high left ventricular myocardial index and low left ventricular ejection fraction are related to LA enlargement [21]. Most of these conditions are also the risk factors for stroke, and they may modify its course.

AF with its co-morbidities has a negative impact on the prognosis for patients with stroke. The most common comorbidities are heart diseases and atherosclerosis of the arteries supplying brain structures. In the present study, patients with AF - compared to those without arrhythmia were more often burdened with diseases that increase the risk of stroke, as expressed by a higher CHA2DS2VASc score used for stratification ( $4.3 \pm 2.0$ vs. $3.6 \pm 1.6 ; p=0.0093)$.

Age is an independent risk factor both for stroke and AF; moreover, it is a prognostic factor in patients with stroke. It was also shown that it has hypercoagulable and prothrombotic effects in patients with $\mathrm{AF}[22,23]$. It is worth noting that some biochemical and vital parameters as abnormal troponin or blood glucose concentration, body temperature besides arterial hypertension and neurological status proved to be an independent factor of post-stroke disability [24-26]. The female sex in this study was associated with a worse prognosis in terms of the post-stroke functional status of patients. Varied responses to thrombolytic therapy in stroke and post-stroke prognosis have been repeatedly discussed in the literature. Despite some disparity in the conclusions, the majority of investigators believe that the female sex is associated with more severe consequences of stroke, and that the use of alteplase (for the benefit of women) eliminates gender-related differences $[27,28]$.

The present study, just like the VISTA meta-analysis, presented no significant differences in the incidence of iatrogenic bleeding after rt-PA in patients with AF compared to patients without arrhythmia. In accordance with the presented results, increasing the NIHSS value by 1 is related to a 1.2-fold increased risk of intracranial bleeding. Neurological status on the first day by NIHSS turned out to be an independent risk factor for unfavorable prognosis (just like 
reduced $\mathrm{EF}$ ) and death (again CHA2DS2VASc $\geq 3$ points). A neurological status estimation based on NIHSS, just like clinical profile evaluation by CHA2DS2VASc in this group of patients, is one of the key prognostic parameters for the functioning of post-stroke patients and for death $[16,29,30]$.

Most probably, there are a few factors related to AF which modify the acute phase of stroke. Patients with AF may have some past thrombi that undergo fragmentation under the influence of alteplase. It is believed that, compared to the "hard" cholesterol emboli formed with the use of thrombi deposited on atherosclerotic plaques, those AF-associated emboli are more susceptible to rt-PA activity.

Structural and functional abnormalities of the heart accompanying AF most probably are additional decisive factors for the course of the acute phase of stroke and the effect of thrombolytic therapy.

\section{Limitations of the study}

There was no analysis conducted that would consider atrial fibrillation types or the factors present during hospitalization as the ones to affect prognosis, e.g. vital or biochemical parameters, pneumonia.

\section{Conclusions}

The presence of atrial fibrillation worsens the patient's prognosis in terms of the functional status and survival during the acute period of stroke in patients treated with intravenous thrombolysis.

Higher NIHSS and CHA2DS2VASc scores and reduced EF in patients with stroke treated with thrombolysis are the predictors of unfavorable short-term prognosis.

\section{Conflict of interest}

None declared.

\section{Acknowledgement and financial support}

None declared.

\section{RE F E R E N C E S}

[1] The World Bank. Investing in health. Oxford: Oxford University Press; 1993, World Development Report 1993.

[2] Kernan WN, Ovbiagele B, Black HR, Bravata DM, Chimowitz MI, Ezekowitz MD, et al. Guidelines for the prevention of stroke in patients with stroke and transient ischemic attack: a guideline for healthcare professionals from the American Heart Association/American Stroke Association. Stroke 2014;45(July (7)):2160-236. http://dx.doi.org/10.1161/ STR.0000000000000024. Epub 2014 May 1

[3] Young FB, Weir CJ, Lees KR. GAIN International Trial Steering Committee and Investigators. Comparison of the
National Institutes of Health Stroke Scale with disability outcome measures in acute stroke trials. Stroke 2005;36:2187-92.

[4] Weisscher N, Vermeulen M, Roos YB, de Haan RJ. What should be defined as good outcome in stroke trials; a modified Rankin score of 0-1 or 0-2? J Neurol 2008;255:867-74.

[5] Mancia G, De Backer G, Dominiczak A, Cifkova R, Fagard R, Germano G, et al. Guidelines for the management of arterial hypertension: The Task Force for the Management of Arterial Hypertension of the European Society of Hypertension (ESH) and of the European Society of Cardiology (ESC). Eur Heart J 2007;28:1462-536.

[6] American Diabetes Association. Standards of medical care in diabetes - 2013. Diabetes Care 2013;36:11-66.

[7] North American Symptomatic Carotid Endarterectomy Trial Collaborators. Beneficial effect of carotid endarterectomy in symptomatic patients with high-grade carotid stenosis. N Engl J Med 1991;325:445-53.

[8] Wahlgren N, Ahmed N, Davalos A, Ford GA, Grond M, Hacke $\mathrm{W}$, et al. Thrombolysis with alteplase for acute ischaemic stroke in the Safe Implementation of Thrombolysis in Stroke-Monitoring Study (SITS-MOST): an observational study. Lancet 2007;369:275-82.

[9] Gumbinger C, Gruschka P, Bottinger M, Heerlein K, Barrows $\mathrm{R}$, Hacke W, et al. Improved prediction of poor outcome after thrombolysis using conservative definitions of symptomatic hemorrhage. Stroke 2012;43:240-2.

[10] Kimura K, Iguchi Y, Yamashita S, Shibazaki K, Kobayashi K, Inoue T. Atrial fibrillation as an independent predictor for no early recanalization after IV-tPA in acute ischemic stroke. J Neurol Sci 2008;267:57-61.

[11] Kimura K, Iguchi Y, Shibazaki K, Iwanaga T, Yamashita S, Aoki J. IV tPA therapy in acute stroke patients with atrial fibrillation. J Neurol Sci 2009;276:6-8.

[12] Sanak D, Herzig R, Kral M, Bartkova A, Zapletalova J, Hutyra $\mathrm{M}$, et al. Is atrial fibrillation associated with poor outcome after thrombolysis? J Neurol 2010;257:999-1003.

[13] Seet RC, Zhang Y, Wijdicks EF, Rabinstein AA. Relationship between chronic atrial fibrillation and worse outcomes in stroke patients after intravenous thrombolysis. Arch Neurol 2011;68:1454-8.

[14] Generalized efficacy of tPA for acute stroke. Subgroup analysis of the NINDS tPA Stroke Trial. Stroke 1997;28:2119-25.

[15] Bluhmki E, Chamorro A, Davalos A, Machnig T, Sauce C, Wahlgren $\mathrm{N}$, et al. Stroke treatment with alteplase given 3.0-4.5 h after onset of acute ischaemic stroke (ECASS III): additional outcomes and subgroup analysis of a randomised controlled trial. Lancet Neurol 2009;8:1095-102.

[16] Phan TG, Clissold B, Ly J, Ma H, Moran C, Srikanth V, et al. Stroke Severity and Comorbidity Index for Prediction of Mortality after Ischemic Stroke from the Virtual International Stroke Trials Archive-Acute Collaboration. J Stroke Cerebrovasc Dis 2016;25(April (4)):835-42. http://dx. doi.org/10.1016/j.jstrokecerebrovasdis.2015.12.016. Epub 2016 Jan 18

[17] Ntaios G, Vemmou A, Koroboki E, Savvari P, Makaritsis K, Saliaris $\mathrm{M}$, et al. The type of atrial fibrillation is associated with long-term outcome in patients with acute ischemic stroke. Int J Cardiol 2013;167(August (4)):1519-23. http://dx. doi.org/10.1016/j.ijcard.2012.04.131. Epub 2012 May 17

[18] Echocardiographic predictors of stroke in patients with atrial fibrillation: a prospective study of 1066 patients from 3 clinical trials. Arch Intern Med 1998;158(June (12)):1316-20.

[19] Hachet O, Guenancia C, Stamboul K, Daubail B, Richard C, Béjot $Y$, et al. Frequency and predictors of stroke after acute myocardial infarction: specific aspects of in-hospital and postdischarge events. Stroke 2014;45(December (12)):3514- 
20. http://dx.doi.org/10.1161/STROKEAHA.114.006707. Epub 2014 Nov 4

[20] Sanchis L, Montserrat S, Obach V, Cervera Á, Chamorro Á, Vidal $B$, et al. Left atrial function is impaired in some patients with stroke of undetermined etiology: potential implications for evaluation and therapy. Rev Esp Cardiol (Engl Ed) )2016;(March). http://dx.doi.org/10.1016/j. rec.2015.11.033. pii:S1885-5857(16)00028-1 [Epub ahead of print]

[21] Ou Q Chen Y, Yu S, Guo X, Zhao H, Sun Y. Prevalence of left atrial enlargement and its risk factors in general Chinese population. BMC Cardiovasc Disord 2016;16(March (1)):53. http://dx.doi.org/10.1186/s12872-016-0229-z

[22] Conway DS, Heeringa J, Van Der Kuip DA, Chin BS, Hofman A, Witteman JC, et al. Atrial fibrillation and the prothrombotic state in the elderly: the Rotterdam Study. Stroke 2003;34:413-7.

[23] Roldan V, Marin F, Garcia A, Tello-Montoliu A, Lip GY. Is an advanced age an additive risk factor to the prothrombotic or hypercoagulable state in atrial fibrillation? Int J Cardiol 2006;110:265-6.

[24] Wira CR, Rivers E, Martinez-Capolino C, Silver B, Iyer G, Sherwin R, et al. Cardiac complications in acute ischemic stroke. West J Emerg Med 2011;12:414-20.
[25] Koennecke HC, Belz W, Berfelde D, Endres M, Fitzek S, Hamilton F, et al. Factors influencing in-hospital mortality and morbidity in patients treated on a stroke unit. Neurology 2011;77:965-72.

[26] Giuffrida S, Carpinteri G, Modica D, Pennisi M, Catalano A, Bella R, et al. Focal neurological deficit in acute anemia: case reports and review of the literature. World J Neurosci 2013;3:53-6.

[27] Kent D, Buchan A, Hill M. The gender effect in stroke thrombolysis: of CASES, controls, and treatment-effect modification. Neurology 2008;71(September (14)):1080-3.

[28] Kent D, Price L, Ringleb P, Hill MD, Selker HP. Sex-based differences in response to recombinant tissue plasminogen activator in acute ischemic stroke: a pooled analysis of randomized clinical trials. Stroke 2005;36(January (1)):62-5.

[29] Katayama T, Fujiwara N, Tsuruya Y. Factors contributing to left atrial enlargement in adults with normal left ventricular systolic function. J Cardiol 2010;55:196-204.

[30] Tu HT, Campbell BC, Meretoja A, Churilov L, Lees KR, Donnan GA, et al. Pre-stroke CHADS2 and CHA2DS2-VASc scores are useful in stratifying three-month outcomes in patients with and without atrial fibrillation. Cerebrovasc Dis 2013;36(4):273-80. http://dx.doi.org/10.1159/000353670. Epub 2013 Oct 16 